Published in final edited form as:

Curr Mol Pharmacol. 2017 ; 10(1): 3-5. doi:10.2174/1874467209999160114145952.

\title{
Editorial (Part 1)
}

\author{
Saeid Ghavami, PhD [Assistant Professor (Guest Editor)], \\ Department of Human Anatomy and Cell Sciences, Rady College of Medicine, Max Rady Faculty \\ of Health Sciences, University of Manitoba, 130-745 Bannatyne Ave., Winnipeg, MB R3E 0J9 \\ Behzad Yeganeh, PhD [(Guest Editor)], \\ Hospital for Sick Children \& University of Toronto \\ Nicholas J. Kenyon, M.D. [(Guest Editor)], and \\ Gordon Wong Professor of Medicine Chief, Division of Pulmonary, Critical Care, Sleep Medicine, \\ Department of Internal Medicine, Center for Comparative Respiratory Biology and Medicine, \\ University of California, Davis School of Medicine, Davis, CA 95616
}

\author{
Amir A. Zeki, M.D. [(Guest Editor)] \\ Assistant Professor of Medicine, Division of Pulmonary, Critical Care and Sleep Medicine, \\ Department of Internal Medicine, Center for Comparative Respiratory Biology and Medicine, \\ University of California, Davis School of Medicine, Davis, CA 95616
}

The mevalonate (MVA) cascade is also known as the cholesterol biosynthesis pathway. This critical cellular pathway is not only responsible for the biosynthesis of cholesterol but is also the source of several essential intermediate metabolites such as geranylgeranylpyrophosphate (GGPP) and farnesylpyrophosphate (FPP) which are vital for normal cell metabolism and function [1]. As with cholesterol, the isoprenoids GGPP and FPP also play a fundamental role in human disease.

The statin drugs (a.k.a. 'statins') are competitive inhibitors of hydroxyl-methyl-glutaryl coenzyme-A (HMG-CoA) reductase (HMGCR), which converts HMG-CoA into mevalonic acid (or mevalonate). HMGCR is also the rate-limiting step in cholesterol biosynthesis. In eukaryotes, the MVA pathway is the only biochemical mechanism capable of generating the isoprenoids, FPP and GGPP, as well as cholesterol. The chemical structure of statins has an HMG-like moiety that binds to a portion of the HMG-CoA binding site, thus blocking access of the HMG-CoA substrate to the enzymatic active site. This inhibition in turn effectively and directly reduces the rate of MVA production [2].

The statins were first discovered in 1976 when a fungal metabolite isolated from Penicillium citrinium was observed to inhibit HMGCR [3]. Soon after, several different statins were discovered and isolated, and further classified for broader use in the clinical arena. The statins are typically divided into several different classes based on whether they are naturally produced by fungi (type 1; e.g. lovastatin, pravastatin, and simvastatin) or synthetically produced (type 2 ; e.g. atorvastatin, fluvastatin, pi-tavastatin, and rosuvastatin). The conserved HMG-like moiety seen on all statins, is covalently bound to an extended hydrophobic group which stabilizes statin binding to HMGCR. 
While statins are widely known to reduce cholesterol biosynthesis, they also decrease critical MVA cascade intermediates such as FPP, GGPP, and downstream squalene, dolichols, and coenzyme Q10 [4, 5]. Currently, MVA cascade inhibitors such as the statins are considered amongst the safest drugs, and are widely used for the treatment of cardiovascular diseases where they have long-standing established clinical benefits.

We have been investigating the various roles of the MVA cascade in models of respiratory, cardiovascular, and cancer diseases and have contributed to the understanding of how MVA metabolism and the statins participate in the development and treatment of disease [1, 6-12]. Furthermore, there is increasing interest in the diverse applications of MVA cascade inhibitors, and a desire to better understand the role of MVA metabolism in common chronic human diseases.

Hence, in collaboration with "Current Molecular Pharmacology" we have prepared a twopart volume of selected papers that cover various aspects of the MVA cascade as it relates to several prevalent conditions. As Editors, we feel privileged to have world-class clinicians and scientists share their expertise through this unique volume. While not comprehensive of all the diseases that MVA metabolism is known to affect, this volume specifically covers the latest knowledge of how the MVA pathway modulates pathogenic mechanisms relevant to respiratory science, developmental biology, cancer, and neuroscience.

In Part 1 of 2 of this volume, we focus on MVA mechanisms in health and disease with a focus on respiratory disease, immune responses and host defense, and aspects of cancer. Five articles cover these areas in detail with expert commentary from our invited authors. In the first article, Muller et al review the importance of the MVA isoprenoid intermediates FPP and GGPP which are involved in the isoprenylation of many proteins including the small GTPases Ras and Rho families. They focus on Rho, Ras, and Rac isoprenylation and its effects on disease pathogenesis. They also address the essential functions of the MVA cascade and its inhibitors (e.g. statins) in the regulation of inflammatory responses in various disease conditions. They finish by discussing the inflammatory effects of MVA and address the clinical implications of mevalonate kinase deficiency, a congenital human disease that sheds light on the fundamental roles of MVA metabolism in human health.

The second article by Hashemi et al. reviews the significance of the MVA cascade in different tissues in health and disease. They provide a valuable review on the regulation of cholesterol metabolism in humans with special attention to the role of different circulating lipoproteins. This section is followed by a comprehensive review on different functional polymorphisms that affect the MVA cascade in various cancers, cardiovascular diseases, and infectious diseases. Finally, the article briefly addresses the use of different statins in cancer.

The MVA cascade and its isoprenoid intermediates are also involved in many lung diseases including asthma [1,13], chronic obstructive pulmonary diseases (COPD) [1, 14] and lung cancer $[1,15]$, to name only a few common conditions. In the current volume, we have included the following three manuscripts discussing several novel aspects of the MVA cascade in different lung diseases and the potential role of statins for the treatment of asthma and COPD. 
Gabor et al. review the importance of the MVA cascade and cholesterol trafficking in pulmonary host defense. The authors describe how cholesterol metabolism and its trafficking is particularly crucial in the regulation of lung physiology, and they further review how cholesterol trafficking regulates macrophages and the innate immune response in the lung. They also highlight the importance of cholesterol trafficking in lung antiviral responses as well as the potential roles of statins as antiviral and antibacterial therapeutic strategies. In addition, they elaborate in detail on the involvement of cholesterol biosynthesis in pulmonary innate immunity as a fundamental process, and discuss the implications of such mechanisms for lung health and disease.

R.P Young et al. have provided a thorough review of how the MVA cascade intersects with immunomodulation in COPD and COPD-related lung cancer. They provide evidence that smoke-induced lung inflammation and genetic susceptibility are involved in both COPD and lung cancer. The regulatory role of the Rho GTPase protein on pro-inflammatory signaling mechanisms is also highlighted. The authors discuss how these proteins could modulate innate immune responses and inflammation in COPD and lung cancer. This review also presents the latest pre-clinical and clinical data on the role of statins and their effects on the MVA pathway in human lung cancer. The possible chemo-preventive effects of statins on lung cancer are addressed, particularly in patients with COPD where inflammation is increased and the risk of lung cancer is greatest.

For a broader view that encompasses both asthma and COPD, N.C. Thomson provides a detailed overview on the latest clinical studies on statins in patients with asthma or COPD. In this article, the clinical effects of statin therapy in asthma and their local airway antiinflammatory effects are reviewed. Dr. Thomson also discusses the possible clinical benefits of statin therapy in the subgroup of COPD patients with cardiovascular diseases given their higher disease risk profile. This provides a rich context for the potential clinical utility of the HMGCR inhibitors in the treatment of chronic airway disorders.

Collectively, this volume of ten articles organized in two parts addresses the basic science that underlies the clinical importance of the MVA cascade and how targeting this important pathway may offer novel therapies for various conditions. The underlying premise is that many of these chronic diseases partially share a common endotype in the form of MVA metabolism that underlies many of the pathologies observed in different diseases.

Additionally, this volume further provides an updated platform where both basic and clinical scientists can access the latest developments in cholesterol/isoprenoid metabolism, and apply this knowledge in the service of drug development and the treatment of patients.

\section{References}

1. Yeganeh B, Wiechec E, Ande SR, Sharma P, Moghadam AR, Post M, Freed DH, Hashemi M, Shojaei S, Zeki AA, Ghavami S. Targeting the mevalonate cascade as a new therapeutic approach in heart disease, cancer and pulmonary disease. Pharmacol Ther. 2014; 143(1):87-110. [PubMed: 24582968]

2. Istvan ES, Deisenhofer J. Structural mechanism for statin inhibition of HMG-CoA reductase. Science. 2001; 292(5519):1160-1164. [PubMed: 11349148] 
3. Endo A, Kuroda M, Tsujita Y. ML-236A, ML-236B, and ML-236C, new inhibitors of cholesterogenesis produced by Penicillium citrinium. J Antibiot (Tokyo). 1976; 29(12):1346-1348. [PubMed: 1010803]

4. Alegret M, Silvestre JS. Pleiotropic effects of statins and related pharmacological experimental approaches. Timely Top Med Cardiovasc Dis. 2007; 11:E10. [PubMed: 17473899]

5. Alegret M, Silvestre JS. Pleiotropic effects of statins and related pharmacological experimental approaches. Methods Find Exp. Clin Pharmacol. 2006; 28(9):627-656.

6. Zeki AA, Bratt JM, Chang KY, Franzi LM, Ott S, Silveria M, Fiehn O, Last JA, Kenyon NJ. Intratracheal instillation of pravastatin for the treatment of murine allergic asthma: a lung-targeted approach to deliver statins. Physiol Rep. 2015; 3(5):e12352. [PubMed: 25969462]

7. Zeki AA, Franzi L, Last J, Kenyon NJ. Simvastatin inhibits airway hyperreactivity: implications for the mevalonate pathway and beyond. Am J Respir Crit Care Med. 2009; 180(8):731-740. [PubMed: 19608720]

8. Zeki AA, Kenyon NJ, Goldkorn T. Statin drugs, metabolic pathways, and asthma: a therapeutic opportunity needing further research. Drug Me-tab Lett. 2011; 5(1):40-44.

9. Zeki AA, Kenyon NJ, Yoneda K, Louie S. The adult asthmatic. Clin Rev Allergy Immunol. 2012; 43(1-2):138-155. [PubMed: 21424682]

10. Ghavami S, Sharma P, Yeganeh B, Ojo OO, Jha A, Mutawe MM, Kashani HH, Los MJ, Klonisch T, Unruh H, Halayko AJ. Airway mesenchymal cell death by mevalonate cascade inhibition: integration of autophagy, unfolded protein response and apoptosis focusing on Bcl2 family proteins. Biochim Biophys Acta. 2014; 1843(7):1259-1271. [PubMed: 24637330]

11. Ghavami S, Yeganeh B, Stelmack GL, Kashani HH, Sharma P, Cunnington R, Rattan S, Bathe K, Klonisch T, Dixon IM, Freed DH, Halayko AJ. Apoptosis, autophagy and ER stress in mevalonate cascade inhibition-induced cell death of human atrial fibroblasts. Cell Death Dis. 2012; 3:e330. [PubMed: 22717585]

12. Schaafsma D, Dueck G, Ghavami S, Kroeker A, Mutawe MM, Hauff K, Xu FY, McNeill KD, Unruh H, Hatch GM, Halayko AJ. The mevalonate cascade as a target to suppress extracellular matrix synthesis by human airway smooth muscle. Am J Respir Cell Mol Biol. 2011; 44(3):394403. [PubMed: 20463291]

13. Schaafsma D, McNeill KD, Mutawe MM, Ghavami S, Unruh H, Jacques E, Laviolette M, Chakir J, Halayko AJ. Simvastatin inhibits TGFbeta1-induced fibronectin in human airway fibroblasts. Respir Res. 2011; 12:113. [PubMed: 21864337]

14. Marin L, Colombo P, Bebawy M, Young PM, Traini D. Chronic obstructive pulmonary disease: patho-physiology, current methods of treatment and the potential for simvastatin in disease management. Expert Opin Drug Deliv. 2011; 8(9):1205-1220. [PubMed: 21615218]

15. Ghavami S, Mutawe MM, Sharma P, Yeganeh B, McNeill KD, Klonisch T, Unruh H, Kashani HH, Schaafsma D, Los M, Halayko AJ. Mevalonate cascade regulation of airway mesenchymal cell autophagy and apoptosis: a dual role for p53. PLoS One. 2011; 6(1):e16523. [PubMed: 21304979] 